\title{
Neutron electric dipole moment from lattice QCD
}

\section{Eigo Shintani ${ }^{* \dagger}$}

Graduate School of Pure and Applied Sciences, University of Tsukuba

\section{S. Aoki}

Graduate School of Pure and Applied Sciences, University of Tsukuba, and Riken BNL Research Center, Brookhaven National Laboratory

\section{N. Ishizuka, Y. Kuramashi, A. Ukawa, T. Yoshié}

Graduate School of Pure and Applied Sciences, University of Tsukuba and Center for Computational Sciences, University of Tsukuba

\section{K. Kanaya}

Graduate School of Pure and Applied Sciences, University of Tsukuba

\section{Y. Kikukawa}

Department of Physics, Nagoya University, , Nagoya 464-8602, Japan

\section{Okawa}

Department of Physics, Hiroshima University

We carry out a feasibility study toward a lattice QCD calculation of the neutron electric dipole moment (NEDM) in the presence of the $\theta$ term using two different approaches. In the first method, we calculate the $\mathrm{CP}$-odd electromagnetic form factor $F_{3}$, which becomes the NEDM in the zero momentum transfer limit. At the first order in $\theta$, we derive a formula connecting the lattice three-point function to the $\mathrm{CP}$-odd electromagnetic form factor. In the second method we directly extract the NEDM from the energy difference between spin-up and spin-down neutron states in the presence of a constant electric field, without expanding a small but non-zero $\theta$. We test both approaches numerically, employing the domain-wall quark action with the RG improved gauge action in quenched QCD at $a^{-1} \simeq 2 \mathrm{GeV}$ on a $16^{3} \times 32 \times 16$ lattice, and further applying the second method to the clover quark action at a similar lattice spacing and nucleon mass. We obtain good signals from both approaches. In particular the second method works well with both fermion formulations.

XXIIIrd International Symposium on Lattice Field Theory

25-30 July 2005

Trinity College, Dublin, Ireland

\footnotetext{
${ }^{*}$ Speaker.

†shintani@ het.ph.tsukuba.ac.jp
} 


\section{Introduction}

One of the most stringent constraints on possible violation of parity and time-reversal symmetry in the strong interaction comes from the measurement of the electric dipole moment (EDM) for neutron (NEDM) $\vec{d}_{n}$ and proton (PEDM) $\vec{d}_{p}$. The current upper bound is given by $\left|\vec{d}_{n}\right|<$ $6.3 \times 10^{-26} e \cdot \mathrm{cm}\left(90 \%\right.$ C.L.)[2] and $\left|\vec{d}_{p}\right|<5.4 \times 10^{-24} e \cdot \mathrm{cm}[3]$. On the other hand, QCD allows a gauge invariant renormalizable CP-odd $\theta$ term in the Lagrangian. Crude model estimations $[4,5]$ and the experimental bound on $\left|\vec{d}_{n}\right|$ yield a very stringent bound $\theta \leq O\left(10^{-10}\right)$.

Current model estimates give not only an order of magnitude different results on $\vec{d}_{n}$ but also differ even in its sign. Clearly a first principles determination of $\vec{d}_{n}$ from lattice QCD is required to determine the value of $\theta$, if a non-zero value is found for NEDM in future experiments. Indeed serious attempts toward lattice QCD calculations of NEDM have just been started recently[1, 7, 8]. In this report we present our results on NEDM calculations based on two different methods, one of which has already been published[1].

\section{NEDM from the electromagnetic form factor}

\subsection{Formulation}

Let us consider the electromagnetic form factor of the nucleon defined by

$$
\left\langle N(\vec{p}, s)\left|J_{\mu}^{\mathrm{EM}}\right| N\left(\vec{p}^{\prime}, s^{\prime}\right)\right\rangle=\bar{u}(\vec{p}, s)\left[\frac{F_{3}\left(q^{2}\right)}{2 m_{N}} q_{\nu} \sigma_{\mu \nu} \gamma_{5}+\cdots\right] u\left(\vec{p}^{\prime}, s^{\prime}\right),
$$

where $J_{\mu}^{\mathrm{EM}}$ is the electromagnetic current, $q=p-p^{\prime}$ is the momentum transfer, $|N(\vec{p}, s)\rangle$ is the on-shell nucleon state with momentum $\vec{p}$, energy $p_{0}=\sqrt{m_{N}^{2}+\vec{p}^{2}}$ and helicity $s$. On the righthand side we explicitly write down the form factor $F_{3}$ only, which is related to NEDM as $\left|\vec{d}_{n}\right|=$ $\lim _{q^{2} \rightarrow 0} F_{3}\left(q^{2}\right) / 2 m_{N}=F_{3}(0) / 2 m_{N}$.

The electromagnetic form factor can be extracted from the following three-point correlation function:

$$
\begin{aligned}
& G_{N J_{\mu} N}^{\theta}(q, t, \tau) \equiv{ }_{\theta}\left\langle N(\vec{p}, t) J_{\mu}^{\mathrm{EM}}(\vec{q}, \tau) \bar{N}\left(\vec{p}^{\prime}, 0\right)\right\rangle_{\theta} \\
& =e^{-E_{N^{\theta}}(t-\tau)} e^{-E_{N \theta^{\theta}}^{\prime}} \sum_{s, s^{\prime}} u_{N}^{\theta}(\vec{p}, s) \bar{u}_{N}^{\theta}(\vec{p}, s) W_{\mu}^{\theta}(q) u_{N}^{\theta}\left(\vec{p}^{\prime}, s^{\prime}\right) \bar{u}_{N}^{\theta}\left(\vec{p}^{\prime}, s^{\prime}\right)+\cdots, \\
& W_{\mu}^{\theta}(q)=\gamma_{\mu} F_{1}\left(q^{2}\right)+\frac{F_{2}\left(q^{2}\right)}{2 m_{N}} q_{\nu} \sigma_{\mu v}+i \theta\left(\frac{F_{3}\left(q^{2}\right)}{2 m_{N}} q_{\nu} \gamma_{5} \sigma_{\mu \nu}+F_{A}\left(q^{2}\right)\left(q_{\mu} \phi-q^{2} \gamma_{\mu}\right) \gamma_{5}\right)+O\left(\theta^{2}\right)
\end{aligned}
$$

where $N(\vec{p}, t)$ and $\bar{N}(\vec{p}, t)$ are the interpolating fields of the nucleon at time $t$, and $F_{1}$ and $F_{2}$ are CP-even form factors while $F_{3}$ and $F_{A}$ are CP-odd. The on-shell spinor $u_{N}^{\theta}$ satisfies

$$
\sum_{s} u_{N}^{\theta}(\vec{p}, s) \bar{u}_{N}^{\theta}(\vec{p}, s)=Z_{N}^{\theta} \frac{-i \gamma \cdot p+m_{N^{\theta}} e^{i f_{N}(\theta) \gamma_{5}}}{2 E_{N^{\theta}}}=Z_{N} \frac{-i \gamma \cdot p+m_{N}}{2 E_{N}}+i \theta Z_{N} \frac{f_{N}^{1} m_{N}}{2 E_{N}} \gamma_{5}
$$

and for small $\theta$ we have an expansion of the forms $m_{N^{\theta}}=m_{N}+O\left(\theta^{2}\right), Z_{N}^{\theta}=Z_{N}+O\left(\theta^{2}\right)$ and $f_{N}(\theta)=f_{N}^{1} \theta+O\left(\theta^{3}\right)$. 
In lattice simulations, the three-point function is calculated as

$$
G_{N J_{\mu} N}^{\theta}(q, t, \tau)=\int \mathscr{D} U \mathscr{D} \bar{\psi} \mathscr{D} \psi e^{S_{\mathrm{QCD}}+i \theta Q}=G_{N J_{\mu} N}(q, t, \tau)+i \theta G_{N_{\mu} N}^{Q}(q, t, \tau)+O\left(\theta^{2}\right) .
$$

Comparing eq.(2.2) with eq.(2.5), we obtain two independent formulae which allow an extraction of $F_{3}$. They are given by

$$
\begin{aligned}
\operatorname{tr} & {\left[G_{N J_{4} N}^{Q}(q, t, \tau) \Gamma_{4} \gamma_{5}\right]=\left|Z_{N}\right|^{2} e^{-E_{N}(t-\tau)} e^{-E_{N}^{\prime} t} } \\
& \times\left[\frac{\vec{q}^{2}}{2 E_{N} m_{N}} F_{3}\left(q^{2}\right)+\left(\frac{E_{N}+m_{N}}{2 E_{N}} F_{1}\left(q^{2}\right)+\frac{\vec{q}^{2}}{4 m_{N} E_{N}} F_{2}\left(q^{2}\right)\right) f_{N}^{1}\right], \\
\operatorname{tr} & {\left[G_{N J_{4} N}^{Q}(q, t, \tau) i \Gamma_{4} \gamma_{5} \gamma_{i}\right]=\left|Z_{N}\right|^{2} e^{-E_{N}(t-\tau)} e^{-E_{N}^{\prime} t} } \\
& \times\left[-\frac{E_{N}+m_{N}}{2 E_{N} m_{N}} q_{i} F_{3}\left(q^{2}\right)+\left(-\frac{q_{i}}{2 E_{N}} F_{1}\left(q^{2}\right)-\frac{q_{i}\left(E_{N}+3 m_{N}\right)}{4 m_{N} E_{N}} F_{2}\left(q^{2}\right)\right) f_{N}^{1}\right],
\end{aligned}
$$

where $\Gamma_{4}=\left(1+\gamma_{4}\right) / 2$. The coefficient $f_{N}^{1}$, which we need to subtract off the mixing terms in the above formulae, can be extracted from the two-point correlation function as

$$
\langle N(p, s) \bar{N}(p, s) Q\rangle=\left|Z_{N}\right|^{2} e^{-E_{N} t} \frac{f_{N}^{1} m_{N}}{2 E_{N}} \gamma_{5},
$$

and $F_{1}$ and $F_{2}$ can be obtained from $G_{N J_{\mu} N}$.

\subsection{Numerical result}

We calculate two- and three-point functions on 730 quenched gauge configurations generated with the RG-improved gauge action at $\beta=2.6$, corresponding to $a^{-1}=1.81$ (4) $\mathrm{GeV}$ determined from $\rho$ meson mass, on a $16^{3} \times 32$ lattice. For the quark action, we employ the domain-wall fermion with the fifth dimension length $N_{s}=16$ and the domain-wall height $M=1.8$, taking the quark mass of $m_{f}=0.03$ corresponding to $m_{P S} / m_{V}=0.63$. The topological charge $Q$ is calculated with the cooling method with an $O\left(a^{2}\right)$ improved definition. As shown in ref.[1], 730 configurations are sufficient to extract the CP-odd phase parameter $f_{N}^{1}$ from the two-point correlation function, and we obtain $f_{N}^{1}=-0.247(17)$.

Subtracting the contribution composed of $F_{1}, F_{2}, f_{N}^{1}$ from the three-point correlation function, we obtain two independent estimates for $F_{3}$ according to eqs.(2.6) and (2.7), which are plotted in Fig. 1 as a function of $t$ for $\tau=6$ being fixed. The two estimates agree at large enough $t$, demonstrating the correctness of our formulae. After fitting the results from eq.(2.6) at $12 \leq t \leq 15$, we obtain

$$
F_{3}\left(q^{2} \simeq 0.58 \mathrm{GeV}^{2}\right) / 2 m_{N}=\left\{\begin{array}{l}
-0.024(5) e \cdot \mathrm{fm} \text { neutron } \\
+0.021(6) e \cdot \mathrm{fm} \text { proton }
\end{array} .\right.
$$

\section{NEDM from the energy shift due to the electric field}

In the previous section, using the quenched lattice QCD calculation, we successfully obtained the signal for the CP-odd form factor $F_{3}$. However, calculating NEDM still requires the limit $q^{2} \rightarrow 0$, which is not so easy in lattice QCD since momenta can be varied only discretely in a finite box and the statistical error increases as the momentum increases. Therefore, we have investigated an alternative method proposed in ref.[9], which does not require the $q^{2} \rightarrow 0$ extrapolation. 

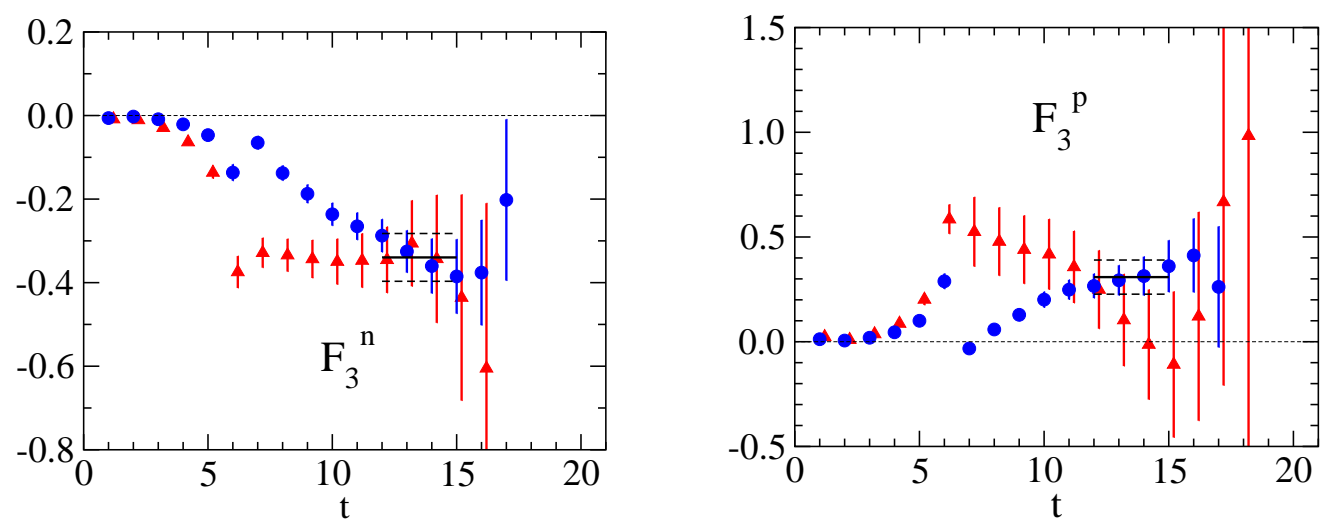

Figure 1: The $F_{3}$ term for neutron (left) and proton (right) as a function of $t$ with the current inserted at $\tau=6$. Circles represent the result with $\Gamma_{4} \gamma_{5}$ projection in eq.(2.6), while triangles that with $\Gamma_{4} \gamma_{5} \gamma_{j}$ in eq.(2.7), averaged over $j=1,2,3$. Straight lines show the fitting results for the former.

\subsection{Formulation}

A non-zero NEDM generates an energy shift of the nucleon in the presence of a uniform and static electric field $\vec{E}$. For $\vec{E}=(0,0, E)$, for example, the energy difference between the spin-up state $N_{\uparrow}$ and the spin-down state $N_{\downarrow}$ takes the form $m_{N_{\uparrow}^{\theta}}(E)-m_{N_{\downarrow}^{\theta}}(E)=d_{n}^{\theta} E+O\left(E^{3}\right)$.

We include a uniform electric field by the substitution $U_{3}(x) \rightarrow U_{3}(x) e^{q E t}$, where the quark charge $q$ is $2 / 3$ for the up quark or $-1 / 3$ for the down quark. This substitution, necessary for a real electric field in Minkowski space, violates the periodicity in the t-direction. In order to minimize its effect at the boundary, we have to take the electric field as small as possible. Choosing appropriate spin components in the neutron propagator, we have

$$
R(E, \theta, t) \equiv\left[\left\langle N_{1} \bar{N}_{1}\right\rangle_{\theta}(E, t)\right] /\left[\left\langle N_{2} \bar{N}_{2}\right\rangle_{\theta}(E, t)\right]=Z e^{-d_{n}^{\theta} E t+O\left(E^{3}\right)}+\cdots
$$

where

$$
\left\langle N_{\alpha} \bar{N}_{\alpha}\right\rangle_{\theta}(E, t)=\left\langle N_{\alpha}(t) \bar{N}_{\alpha}(0) e^{i \theta Q}\right\rangle_{E}
$$

and $\langle\mathscr{O}\rangle_{E}$ represents the vacuum expectation value of $\mathscr{O}$ without the $\theta$ term but with $E$. Since the neutron propagator calculated at $\theta=0$ is averaged with the weight $e^{i \theta Q}$ ("reweighting") to obtain the $\theta$-average, a good sampling of the topological charge $Q$ is crucially important for a success of this method.

\subsection{Numerical results with the domain-wall fermion}

We take the same simulation parameters as in the previous section except for a heavier quark mass, $m_{f}=0.12$, corresponding to $m_{N} \simeq 2.2 \mathrm{GeV}$. The number of configurations is increased to 1000 .

On the left of Fig.2 the ratio eq.(3.1) is plotted as a function of $t$ for $E= \pm 0.004$ and $\theta=0.1$ (circles), and for $E=0.004$ and $\theta=0$ (bursts). The behavior of the ratio is consistent with the expectation that the exponent depends on the sign of $E$ and that the ratio is unity for $\theta=0$. On the right in Fig. 2 we show the effective mass of the ratio, from which we determine the fitting range to be $5 \leq t \leq 9$. 

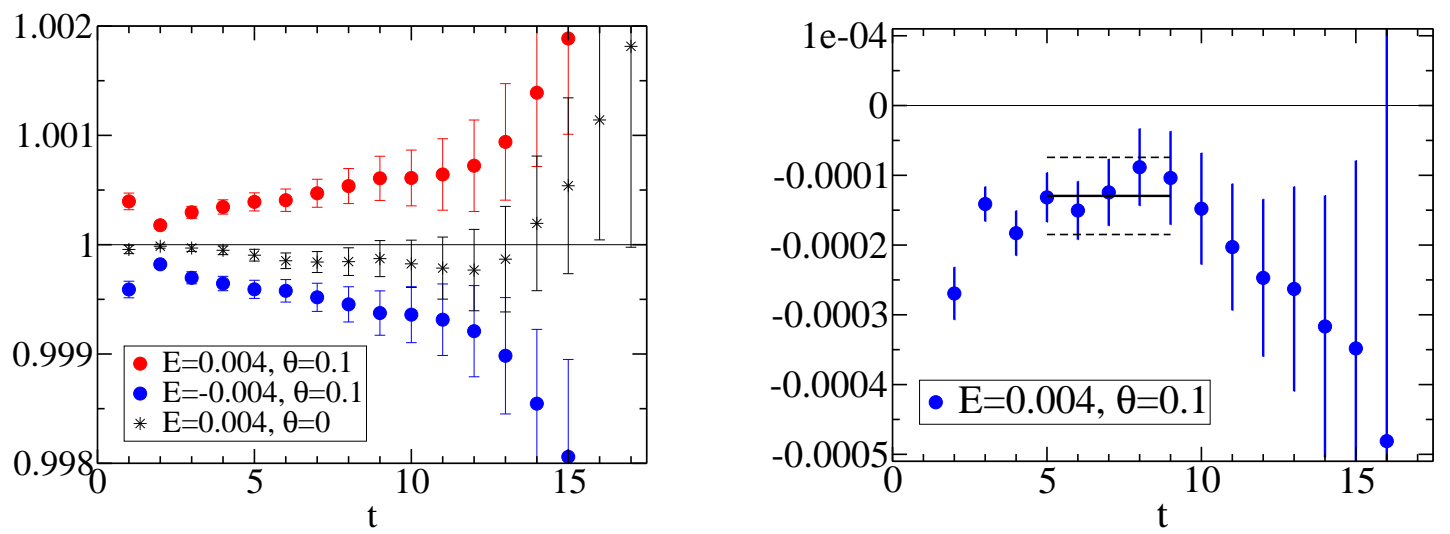

Figure 2: (Left) The ratio eq.(3.1) as a function of $t$ for $E= \pm 0.004$ and $\theta=0.1$ (circles), and for $E=0.004$ and $\theta=0$ (bursts). (Right) An effective mass plot of the ratio.

As is seen on the left of Fig.3, the exponent of $R(E, \theta, t)$ has a linear behavior in $E$ as expected. Finally, in right of Fig.3, $d_{n}^{\theta}$ is plotted as a function of $\theta$. A slope obtained by a linear fit becomes

$$
d_{n}^{\theta} / \theta=-0.0183(60) e \cdot \mathrm{fm}, \quad \mathrm{d}_{\mathrm{p}}^{\theta} / \theta=0.0176(72) \mathrm{e} \cdot \mathrm{fm} .
$$

These values are smaller than those obtained with the CP-odd form factor at $q^{2} \simeq 0.58 \mathrm{GeV}^{2}$ ( eq.(2.9) ). Note, however, that the quark mass is a factor four larger in the present calculation.

\subsection{Preliminary results with the clover fermion}

Since the Wilson-clover fermion action requires much less computational costs, and there exists a large number of full QCD configurations generated with this action, it is important to test if the method of energy difference works with it. We calculate the ratio $R(E, \theta, t)$ using the gauge configurations generated with the same parameters, and choosing the hopping parameter $K=0.1320$ which yields a nucleon mass similar to the domain-wall case. In Fig.4 we present our preliminary result for the effective mass. It looks very similar to the one with the domain-wall action. Our results suggest that the difference of the two fermion actions does not affect the success of this method.
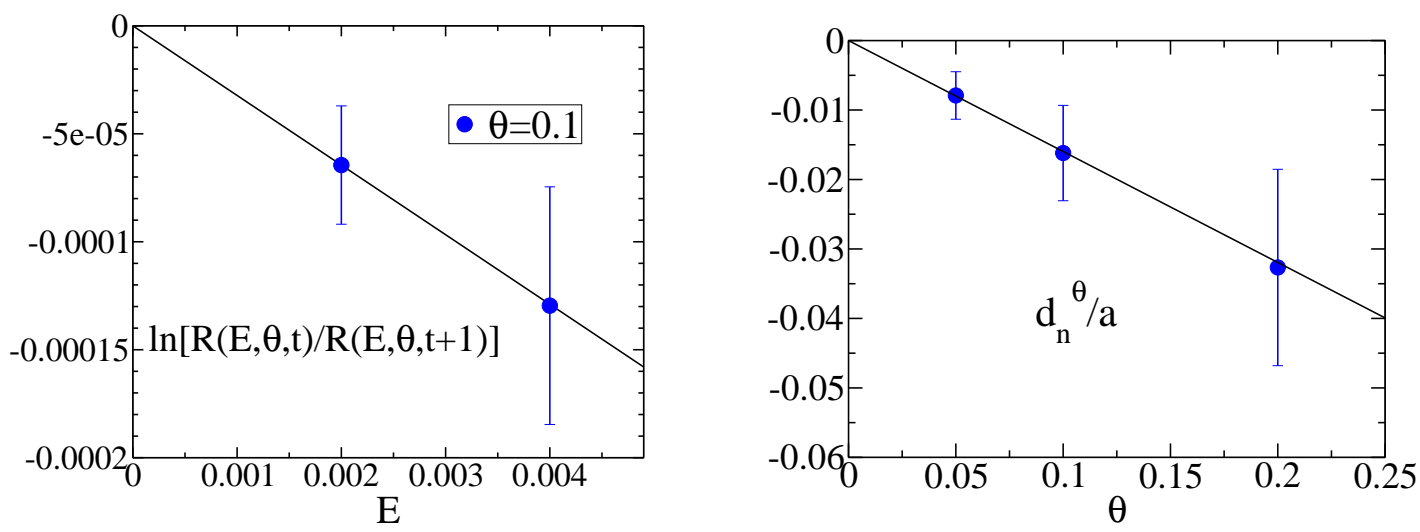

Figure 3: (Left) $E$ dependence the ratio $R(t, E, \theta)$. (Right) $\theta$ dependence of NEDM over lattice spacing $a$. 


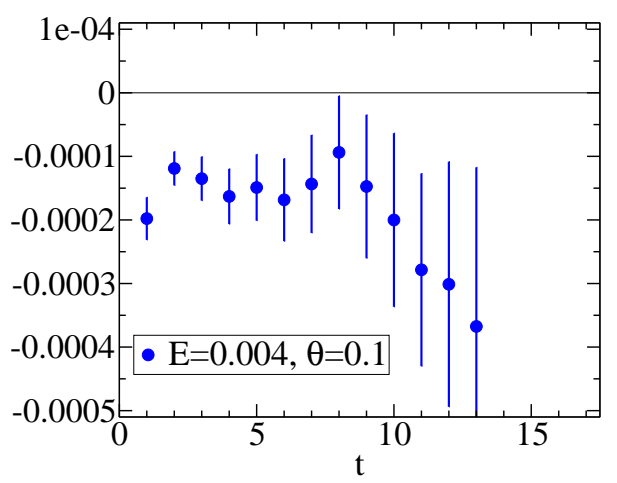

Figure 4: An effective mass plot as Fig. 2 with the clover fermion at $K=0.1320, \theta=0.1$ and $E=0.004$.

\section{Conclusion and outlook}

In this report we have proposed and tested two different approaches for the calculation of NEDM on the lattice. In the first method the CP-odd electromagnetic form factor is employed, while in the second one the energy shift is calculated in the presence of an electric field. We have shown that both methods work well with the domain-wall quark action. Furthermore we have demonstrated that the second method also works with the clover quark action. We are now calculating NEDM at lighter quark masses with the clover quark action in quenched QCD, in order to see how errors increase as the quark mass decreases. Our final target is the calculation of NEDM on full QCD configurations generated with the clover quark action by the CP-PACS collaboration.

This work is supported in part by Grant-in-Aid of the Ministry of Education (Nos. 13640260, 15204015, 15540251, 15740134, 15740165, 16028201, 16540228, 17540249, 17540259 ).

\section{References}

[1] E. Shintani, et al., Phys. Rev. D72, 014504 (2005) [hep-lat / 0505022 ].

[2] P. G. Harris et al., Phys. Rev. Lett. 82, 904 (1999).

[3] V. F. Dmitriev, R. A. Sen'kov, Phys. Rev. Lett. 91, 212303 (2003) [nucl-th/ 0306050 ].

[4] R. J. Crewther, P. Di Vecchia, G. Veneziano, E. Witten, Phys. Lett. B88, (1979) 123; erratum, ibid. B91, 487 (1980); P. Di Vecchia, Acta Phys. Austriaca Suppl. 22, 341 (1980).

[5] M. Pospelov, A. Ritz, Nucl. Phys. B558, 243 (1999) [hep-ph/9903553]; Nucl. Phys. B573, 177 (2000) [hep-ph/9908508]; Chuan-Tsung Chan, E. M. Henley, T. Meissner, [hep-ph/9905317]; M. Pospelov, A. Ritz, Phys. Rev. Lett. 83, 2526 (1999) [hep-ph/ 9904483 ]; Phys. Rev. D63, 073015 (2001) [hep-ph/ 01003 7]; B. Borasoy, Phys. Rev. D61, 114017 (2000) [hep-ph/ 0004011$]$.

[6] R. D. Peccei, H. R. Quinn, Phys. Rev. Lett. 38, 1440 (1977); R. D. Peccei, Adv. Ser. Direct. High Energy Phys. 3, 503-551 (1989).

[7] P. Faccioli, D. Guadagnoli, S. Simula, Phys. Rev. D70, 074017 (2004) [hep-ph / 0406336 ].

[8] F. Berruto, T. Blum, K. Orginos, A. Soni, Nucl. Phys. B(Proc. Suppl.)140, 411 (2005) [hep-lat/ 0411003$]$; T. Blum, talk presented at Lattice 2005, Dublin, July 2005

[9] S. Aoki, A. Gocksch, Phys. Rev. Lett. 63, 1125 (1989); erratum, ibid. 65, 1172 (1990); S. Aoki, A. Gocksch, A. V. Manohar, S. R. Sharpe, Phys. Rev. Lett. 65, 1092 (1990). 